\title{
A. Voegele, Morales de la fiction de La Fontaine à Sartre
}

\section{Germana Berlantini}

\section{(2) OpenEdition}

\section{Journals}

\section{Edizione digitale}

URL: http://journals.openedition.org/studifrancesi/10636

DOI: 10.4000/studifrancesi. 10636

ISSN: 2427-5856

\section{Editore}

Rosenberg \& Sellier

\section{Edizione cartacea}

Data di pubblicazione: 1 décembre 2017

Paginazione: 602-603

ISSN: 0039-2944

\section{Notizia bibliografica digitale}

Germana Berlantini, «A. Voegele, Morales de la fiction de La Fontaine à Sartre», Studi Francesi [Online], 183 (LXI | III) | 2017, online dal 01 février 2018, consultato il 25 janvier 2021. URL: http:// journals.openedition.org/studifrancesi/10636 ; DOI: https://doi.org/10.4000/studifrancesi.10636

Questo documento è stato generato automaticamente il 25 janvier 2021.

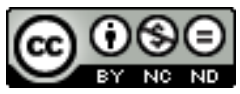

Studi Francesi è distribuita con Licenza Creative Commons Attribuzione - Non commerciale - Non opere derivate 4.0 Internazionale. 


\title{
A. Voegele, Morales de la fiction de La Fontaine à Sartre
}

\author{
Germana Berlantini
}

\section{NOTIZIA}

Augustin VOegele, Morales de la fiction de La Fontaine à Sartre, Paris, Orizons, 154 pp.

1 Il saggio di Augustin vOEGELE si propone come un'analisi delle incursioni della morale nella finzione a partire da incompatibilità e differenze che segnano lo scarto fra i due generi, caratterizzati, il primo, dalla fondazione su di un sapere e sulla volontà di diffonderlo, il secondo, da una relazione di referenza debole se non del tutto assente con la realtà. L'incompatibilità di letteratura e scrittura morale-topos invalso soprattutto fra saggisti, filosofi e moralisti fin dall'epoca di Platone - si radicherebbe dunque nel rifiuto da parte di quest'ultima della deformazione narrativa dei fatti, tanto quanto nell'impossibilità per il testo di finzione di agire sul reale. Il lavoro di Voegele mira a dimostrare come l'innesto di un genere nell'altro avvenga sempre tramite un processo di 'definzionalizzazione' della scrittura letteraria che si attua scavando lacune e deviazioni più tipicamente morali - saggistiche, discorsive o in forma di massima - nel corpo del testo. Per condurre tale indagine, il critico ha selezionato un corpus trasversale, comparatista e diacronico, composto da autori omogenei in quanto a collocazione geografica (europea) e storica (età moderna, dall'epoca classica al xx secolo): La Fontaine, Vauvenargues, Hugo, Tolstoï, Sartre cui sono affiancate le opere di moralisti come La Rochefoucauld, Pascal e Alain. Un primo fattore di coesione fra gli scrittori presi in esame riguarda la nozione di una doppia riforma: in primo luogo diretta, come conversione del lettore, in secondo luogo mediata, come azione sul mondo ad opera del soggetto convertito. Una riforma morale che trova, sul piano biografico, un corrispettivo nelle conversioni religiose o politiche di La Fontaine, Pascal, Hugo e Tolstoï, anch'esse qualificate dal critico di forme di definzionalizzazione, in primo luogo in senso lato per la relazione demistificante con le convinzioni pregresse, in secondo luogo per il rapporto di referenzialità che si stabilisce fra l'opera 
e l'exemplum incarnato dalla vita dell'autore. Infine un tratto trasversale agli scrittori presi in esame è costituito dal loro interesse pedagogico ed educativo, attuato tramite esperienze di insegnamento o teorizzato propugnando ideali e progetti di educazione universale. Elementi di eccentricità rispetto a questo canone pertengono alla figura di Sartre, caratterizzata dalla rottura con ogni forma di morale kantiana o trascendente e dall'apertura a un pluralismo della scelta etica. Tuttavia l'incompiutezza tanto del ciclo romanzesco di Les Chemins de la liberté quanto del progetto saggistico dei Cahiers pour une morale sembra da un lato inficiare la possibilità di fondare teoricamente una simile morale polifonica, dall'altro confutare la tesi di partenza del presente saggio sull'incompatibilità di morale e finzione. L'ipotesi di Voegele è che, nel passaggio della morale, con Sartre, dall'assolutezza alla parzialità, a tale rapporto di conflittualità si sostituisca una compatibilità per l'appunto 'polifonica' fondata sul superamento della gerarchizzazione ontologica di realtà e finzione.

2 Dopo un'introduzione tematica e metodologica (pp. 9-29), il primo capitolo del volume, intitolato «Fiction littéraire et action morale» (pp. 37-75) prende in esame le diverse teorie sulla referenzialità del discorso finzionale, da Searle a Genette, da Jakobson a Tolstoï e Louis Marin. Un problema che riguarda tanto lo statuto ontologico degli oggetti testuali quanto la mancanza di referenzialità propria di ciò che con Genette si può chiamare dizione: l'universo estetico, stilistico, sonoro di un'opera che ne fonda la specifica autotelia artistica. Il rifiuto della finzione da parte dei moralisti si giustificherebbe non solo alla luce del quoziente di deformazione proprio della dizione letteraria ma anche in virtù di una più generale diffidenza nei confronti del linguaggio, cui è contrapposta la superiorità dei fatti. L'esigenza di ancoraggio referenziale propria del pensiero morale si traduce nella sua fondazione su di un sapere positivo e trasmissibile: all'enfasi posta sul momento della conoscenza - della egge morale per Hugo, di sé stessi per Alain, della natura umana per Pascal e così via - si affianca la vocazione propriamente pedagogica alla comunicazione di tale sapere. A partire da queste premesse il rapporto fra i due generi è analizzato induttivamente deducendo da un'ampia casistica storicamente situata, le invarianti capaci di rappresentare in termini più generali le forme di accomodamento reciproco che scrittura morale e scrittura finzionale stabiliscono di volta in volta. Voegele classifica i tipi di definzionalizzazione a seconda del luogo di collocazione-testuale, paratestuale o contestuale-e del momento, cioè della durata degli effetti prodotti che può essere puntuale, definitiva o dar luogo alla sovrapposizione duratura dei due generi. Il secondo capitolo, dedicato a «Les lieux de défictionnalisation» (pp. 77-117), illustra un'ampia casistica tratta dal corpus di riferimento e analizzata in base all'intersezione fra i due assi suddetti e il genere cui l'opera può essere ascritta (allegorico o a tesi, se la durata dei processi di definzionalizzazione è definitiva o produce una sovrapposizione permanente, finzione pura se l'effetto è puntuale). Oltre che in base alle geografie della loro distribuzione, $\mathrm{i}$ processi di definzionalizzazione sono classificati dal critico, nel terzo capitolo del volume («Les types de défictionnalisation», pp.119-139), secondo la modalità in cui si attuano: alla finzione definzionalizzante, che critica una finzione particolare o la dizione in quanto tale, proponendosi come decostruzione in atto del proprio statuto letterario, si affiancano forme di finzione provvisoria o secondaria, che puntano alla propria traduzione in un messaggio morale, di finzione parziale, in cui massime o digressioni morali sono incastonate nel corpo della narrazione, o di finzione prolungata, in cui è alla vita del lettore e al distacco dalla pagina che è demandato il compito di una rielaborazione propriamente morale del testo. Dall'analisi di una 
molteplicità di luoghi testuali emerge dunque il quadro di una complessificata relazione fra $\mathrm{i}$ due termini del problema in esame che sfida le dichiarazioni d'incompatibilità che si è visto abbondare soprattutto in sede teorica. Alla luce di questo bagaglio critico Voegele può trarre le fila della sua analisi sottolineando come, almeno fra gli autori presi in esame, la finzione abbia rappresentato uno strumento di incarnazione per il pensiero morale, capace di veicolare rappresentazioni idealtipiche di situazioni o comportamenti umani giudicate dai loro artefici più efficaci dei loro corrispettivi fattuali o realistici, senza contare come autori quali Sartre e Hugo abbiano attribuito alla finzione in quanto tale una specifica valenza morale, per le condizioni di libertà proprie della creazione letteraria (per il primo) o per la sua capacità di accedere alla conoscenza del volto occulto della natura che sfugge alla ragione e racchiude le possibilità più autentiche di elevazione spirituale (per il secondo). Tuttavia, nella misura in cui il 'piacere del testo' è ammesso da tutti gli scrittori in esame a posteriori, una volta vagliatane la compatibilità col progetto morale che anima l'opera, resta aperta la questione della compatibilità fra fini morali e gratuità letteraria, desiderio di agire sul reale e sovranità - in termini batailliani - della letteratura. Una questione sulla quale il saggio apre, nella «Conclusion» (pp. 143-149) come su una pista di ricerca essenziale per la prosecuzione del campo di studi inaugurato, assieme a un arricchimento del corpus di testi in esame e al vaglio delle evoluzioni subite dal problema morale dopo la fine degli umanismi. 